\title{
Proton-electron bremsstrahlung
}

\author{
E. Haug ${ }^{\star}$ \\ Institut für Astronomie und Astrophysik, Universität Tübingen, 72076 Tübingen, Auf der Morgenstelle 10, Germany
}

Received 16 May 2003 / Accepted 22 May 2003

\begin{abstract}
The collision of energetic protons with free electrons is accompanied by the emission of bremsstrahlung. If the target electrons are approximately at rest, this process is designated electron-proton bremsstrahlung or suprathermal proton bremsstrahlung. The kinematics and the fully relativistic cross section of proton-electron bremsstrahlung in Born approximation is given. The X-ray spectrum produced by protons with a power-law spectrum is calculated for thin and thick targets.
\end{abstract}

Key words. radiation mechanisms: nonthermal - X-rays: general

\section{Introduction}

The galactic and solar cosmic radiation consists largely of energetic (suprathermal) protons. When a beam of these protons is incident on a plasma, appreciable $\mathrm{X}$ - and gamma radiation is produced in collisions with ambient electrons which are approximately at rest. This process is much the same as the normal electron-proton bremsstrahlung except that now the center of momentum of the proton-electron system is virtually that of the energetic proton. Therefore it was designated suprathermal proton bremsstrahlung (Brown 1970; Boldt \& Serlemitsos 1969), inverse bremsstrahlung ${ }^{1}$, or proton-electron bremsstrahlung (PEB, Heristchi 1986). The PEB process was considered to be a potential production mechanism for the diffuse $\gamma$-ray background (Boldt \& Serlemitsos 1969; Brown 1970; Pohl 1998) and for solar flare hard X-rays (Boldt \& Serlemitsos 1969; Emslie \& Brown 1985; Heristchi 1986).

In the nonrelativistic case (proton velocity $v \ll c$ ) the bremsstrahlung produced by a proton of kinetic energy $E$ has the same spectrum as that of an electron of kinetic energy $T=\left(m_{\mathrm{e}} / m_{\mathrm{p}}\right) E$ in collisions with a stationary proton $\left(m_{\mathrm{e}}\right.$ and $m_{\mathrm{p}}$ are the rest masses of electron and proton, respectively). Since, however, the accuracy of the nonrelativistic Bethe-Heitler cross section for bremsstrahlung falls off rapidly at higher energies (Haug 1997) the corresponding PEB cross section is of small value. At relativistic energies, the derivation of the PEB cross section causes more trouble. The usual bremsstrahlung cross section differential in both the energy and angles of the emitted photon has to be transformed to the electron rest frame and to be integrated over the emission solid angle (Brown 1970; Haug

\footnotetext{
* e-mail: haug@tat.physik.uni-tuebingen.de

1 The term "inverse bremsstrahlung" is inappropriate since it may be confused with the quantum-electrodynamical inverse of conventional bremsstrahlung, the photon absorption by a free electron in the Coulomb field of a nucleus.
}

1972). The calculation by means of the Weizsäcker-Williams method (Jones 1971) which is inherently simpler, yields poor results at the high-energy tails of the PEB spectrum (Haug 1972).

In view of the renewed interest in the X-and gamma-ray production through PEB (Dogiel et al. 1998; Pohl 1998; Valinia \& Marshall 1998; Baring et al. 2000) it is worthwile to provide a fully relativistic cross-section formula where nearly all of the angular integrations are performed analytically thus allowing to calculate the X-ray spectra for arbitrary energy distributions of the incident protons with substantially reduced computational expense.

\section{Kinematics of the process}

The variables of the SPB process are displayed in Fig. 1. In the following the proton energy $\epsilon$ (including rest energy) is expressed in units of $m_{\mathrm{p}} c^{2} \approx 938 \mathrm{MeV}$ and the momentum $\boldsymbol{p}$ in units of $m_{\mathrm{p}} c$, whereas the electron energy $\epsilon_{1}$ and the photon energy $k$ are given in units of $m_{\mathrm{e}} c^{2}$, the electron momentum $\boldsymbol{p}_{1}$ and the photon momentum $\boldsymbol{k}$ in units of $m_{\mathrm{e}} c$. Energy and momentum of the outgoing particles are designated by primed quantities. In order to calculate the maximum photon energy, $k_{\text {max }}$, the finite rest mass of the proton has to be taken into account. Otherwise $k_{\max }$ could be greater than the proton kinetic energy $E$ for $E \gg m_{\mathrm{p}} c^{2}$, in contradiction to energy conservation (Heristchi 1986). In an arbitrary frame of reference the conservation of energy and momentum is most conveniently expressed in terms of the four-momenta which are denoted by underlined quantities. Taking into account the different energy units for protons, electrons, and photons the relation reads

$m_{\mathrm{p}} \underline{p}+m_{\mathrm{e}} \underline{p}_{1}-m_{\mathrm{e}} \underline{k}=m_{\mathrm{p}} \underline{p}^{\prime}+m_{\mathrm{e}} \underline{p}_{1}^{\prime}$ 


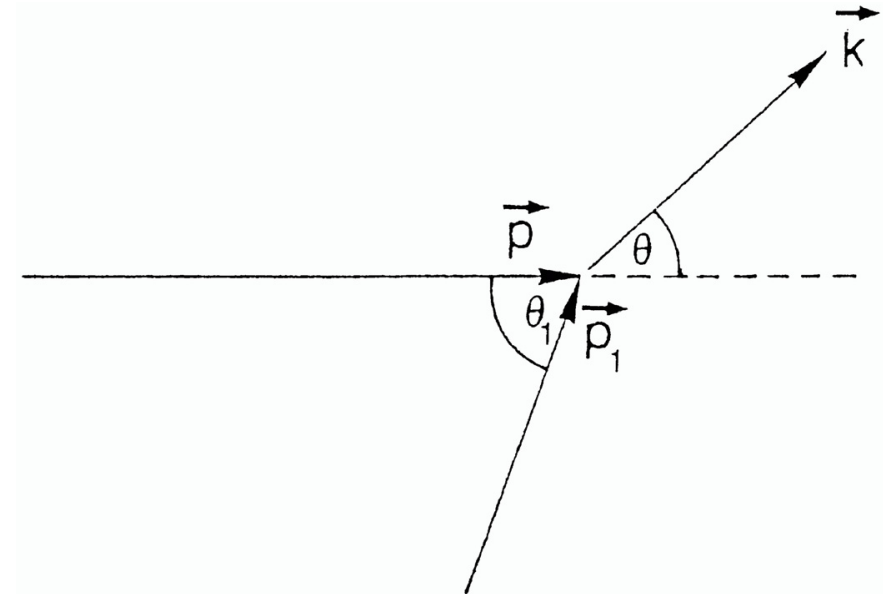

Fig. 1. Momenta and angles in the PEB process.

Squaring this equation yields, utilizing $\underline{p}^{2}=\epsilon^{2}-p^{2}=1$ and $\underline{k}^{2}=0$,

$$
\begin{aligned}
m_{\mathrm{p}}^{2}+m_{\mathrm{e}}^{2}+2 m_{\mathrm{e}} m_{\mathrm{p}}\left[\left(p p_{1}\right)-(p k)\right] & -2 m_{\mathrm{e}}^{2}\left(p_{1} k\right) \\
= & m_{\mathrm{p}}^{2}+m_{\mathrm{e}}^{2}+2 m_{\mathrm{e}} m_{\mathrm{p}}\left(p^{\prime} p_{1}^{\prime}\right)
\end{aligned}
$$

or

$$
m_{\mathrm{p}}\left(p^{\prime} p_{1}^{\prime}\right)=m_{\mathrm{p}}\left(p p_{1}\right)-m_{\mathrm{p}}(p k)-m_{\mathrm{e}}\left(p_{1} k\right)
$$

where $(a b)=a_{0} b_{0}-\boldsymbol{a} \cdot \boldsymbol{b}$ denotes the invariant product of the four-vectors $a=\left(a_{0}, \boldsymbol{a}\right)$ and $b=\left(b_{0}, \boldsymbol{b}\right)$. Since

$\left(p^{\prime} p_{1}^{\prime}\right)=\epsilon^{\prime} \epsilon_{1}^{\prime}-\boldsymbol{p}^{\prime} \cdot \boldsymbol{p}_{1}^{\prime} \geq \epsilon^{\prime} \epsilon_{1}^{\prime}-p^{\prime} p_{1}^{\prime} \geq 1$,

we have

$$
\begin{aligned}
m_{\mathrm{p}}(p k)+m_{\mathrm{e}}\left(p_{1} k\right) & =k\left[m_{\mathrm{p}}(\epsilon-p \cos \theta)+m_{\mathrm{e}}\left(\epsilon_{1}-p_{1} \cos \vartheta\right)\right] \\
& \leq m_{\mathrm{p}}\left[\left(p p_{1}\right)-1\right] \\
& =m_{\mathrm{p}}\left(\epsilon \epsilon_{1}-p p_{1} \cos \theta_{1}-1\right)
\end{aligned}
$$

yielding

$$
k \leq k_{\max }\left(\theta, \theta_{1}, \vartheta\right)=\frac{\epsilon \epsilon_{1}-p p_{1} \cos \theta_{1}-1}{\epsilon-p \cos \theta+\left(m_{\mathrm{e}} / m_{\mathrm{p}}\right)\left(\epsilon_{1}-p_{1} \cos \vartheta\right)},
$$

where $\theta$ and $\vartheta$ is the photon emission angle relative to the incident proton and electron momentum, respectively, and $\theta_{1}$ is the angle between the incident proton and electron (Fig. 1). For fixed values of the energies $\epsilon$ and $\epsilon_{1}$ it is easily seen that the absolute maximum of the photon energy is reached for $\theta=\vartheta=0$ and $\theta_{1}=\pi$, resulting in

$$
k_{\max }\left(\epsilon, \epsilon_{1}\right)=\frac{\epsilon \epsilon_{1}+p p_{1}-1}{\epsilon-p+\left(m_{\mathrm{e}} / m_{\mathrm{p}}\right)\left(\epsilon_{1}-p_{1}\right)} .
$$

If the incident electron is at rest $\left(\epsilon_{1}=1, p_{1}=0\right)$ this reduces to

$$
k_{\max }(\epsilon, 1)=\frac{\epsilon-1}{\epsilon-p+m_{\mathrm{e}} / m_{\mathrm{p}}}<\left(m_{\mathrm{p}} / m_{\mathrm{e}}\right)(\epsilon-1) .
$$

The last inequality implies that the photon energy never exceeds the kinetic energy of the proton, as it should be. Neglecting, however, $m_{\mathrm{e}}$ in the denominator of (8), one obtains

$k_{\max }(\epsilon, 1) \approx(\epsilon-1)(\epsilon+p)$,

which is only valid for $\epsilon \ll m_{\mathrm{p}} /\left(2 m_{\mathrm{e}}\right) \approx 918$, or $E \ll 860 \mathrm{GeV}$.

Solving Eq. (7) for $\epsilon$ and $p$ leads to the minimum energy and momentum, respectively, of the proton required to produce photons of energy $k$. In the following we will restrict to the case that the target electron is at rest in the laboratory system, i.e., $\epsilon_{1}=1, p_{1}=0$. Then we have

$\epsilon_{\min }=\frac{1+k^{2}+2\left(m_{\mathrm{e}} / m_{\mathrm{p}}\right) k+\left(m_{\mathrm{e}} / m_{\mathrm{p}}\right)^{2} k^{2}}{\left[1+\left(m_{\mathrm{e}} / m_{\mathrm{p}}\right) k\right](1-k)+k R}$,

$p_{\min }=k \frac{1+\left(m_{\mathrm{e}} / m_{\mathrm{p}}\right)\left[2-k+\left(m_{\mathrm{e}} / m_{\mathrm{p}}\right) k\right]}{k\left[1+\left(m_{\mathrm{e}} / m_{\mathrm{p}}\right) k\right]+(1-k) R}$,

where

$R=\sqrt{2 k\left(1+m_{\mathrm{e}} / m_{\mathrm{p}}\right)+\left(m_{\mathrm{e}} / m_{\mathrm{p}}\right)^{2} k^{2}}$.

Formula (10) is needed for the calculation of the photon spectra from protons with an energy distribution $f(\epsilon)$ (see Sect. 4).

For $\epsilon \ll m_{\mathrm{p}} / 2 m_{\mathrm{e}}$, Eqs. (10) and (11) reduce to

$\epsilon_{\min } \approx 1+\frac{k}{1+\sqrt{2 k}}, \quad p_{\min } \approx \frac{k+\sqrt{2 k}}{1+\sqrt{2 k}}$.

\section{Cross sections}

We consider the collision of an energetic proton with a stationary electron $\left(p_{1}=0\right)$. Designating the variables in the proton rest system by an asterisk, the invariance of the four-product ( $p k)$ yields

$k^{*}=k(\epsilon-p \cos \theta)$.

The invariance of the doubly differential bremsstrahlung cross section implies

$\frac{\mathrm{d}^{2} \sigma}{\mathrm{d} k \mathrm{~d} \Omega} \mathrm{d} k \mathrm{~d} \Omega=\frac{\mathrm{d}^{2} \sigma^{*}}{\mathrm{~d} k^{*} \mathrm{~d} \Omega^{*}} \mathrm{~d} k^{*} \mathrm{~d} \Omega^{*}$,

where $\mathrm{d} \Omega=\sin \theta \mathrm{d} \theta \mathrm{d} \varphi$ is the element of solid angle of the emitted photon.

Using the relativistic transformation formula for the photon emission angle $\theta$,

$\cos \theta^{*}=\frac{\epsilon \cos \theta-p}{\epsilon-p \cos \theta}$,

one gets

$\frac{\mathrm{d}^{2} \sigma}{\mathrm{d} k \mathrm{~d} \Omega}=\frac{1}{\epsilon-p \cos \theta} \frac{\mathrm{d}^{2} \sigma^{*}}{\mathrm{~d} k^{*} \mathrm{~d} \Omega^{*}}$.

The cross section $\mathrm{d}^{2} \sigma^{*} /\left(\mathrm{d} k^{*} \mathrm{~d} \Omega^{*}\right)$ is the common bremsstrahlung cross section (neglecting proton recoil) where the quantities $\epsilon_{1}^{*}=\epsilon, \epsilon_{2}^{*}=\epsilon_{1}^{*}-k^{*}, k^{*}$, and $\cos \theta^{*}$ are expressed by the corresponding quantities in the rest system of the electron by means of Eqs. (14) and (16). Using 
the fully relativistic bremsstrahlung cross section in Born approximation as given by Sauter (1934) it has the form ${ }^{2}$

$$
\begin{aligned}
\frac{\mathrm{d}^{2} \sigma^{*}}{\mathrm{~d} k^{*} \mathrm{~d} \Omega^{*}} & =\frac{\alpha r_{0}^{2}}{2 \pi} \frac{p_{2}^{*}}{p^{2} k}(\epsilon-p \cos \theta) \\
& \times\left\{\frac{1}{p}\left[\epsilon^{2}+\frac{\epsilon}{\epsilon-p \cos \theta}-2\left(2 \epsilon^{2}+1\right) \cos ^{2} \theta\right]\right. \\
& +k(p-\epsilon \cos \theta)+\frac{p^{2}}{p_{2}^{* 2}+2 k}[k(p-\epsilon \cos \theta)-p] \\
& -\frac{2 p \ln \left(\epsilon_{2}^{*}+p_{2}^{*}\right)}{p_{2}^{*}(\epsilon-p \cos \theta)}+\frac{p}{p_{2}^{*} \sqrt{p_{2}^{* 2}+2 k}} \ln \frac{\sqrt{p_{2}^{* 2}+2 k}+p_{2}^{*}}{\sqrt{p_{2}^{* 2}+2 k}-p_{2}^{*}} \\
& \times\left[1-2 k+\frac{k^{2}}{p_{2}^{* 2}+2 k}\left\{k(\epsilon-p \cos \theta)^{2}+p(\epsilon \cos \theta-p)\right\}\right] \\
& +\frac{1}{p_{2}^{*}} \ln \frac{p\left(p+p_{2}^{*}\right)-\epsilon k(\epsilon-p \cos \theta)}{k(\epsilon-p \cos \theta)} \\
& \times\left[2 \epsilon \sin ^{2} \theta\left\{k(\epsilon-p \cos \theta)\left(3 / p^{2}+1\right)-\epsilon\right\}\right. \\
& +\left(2 \epsilon^{2}-1\right)\left(1+k / p^{2}\right)+2\{\epsilon-k(\epsilon-p \cos \theta)\}^{2} \\
& \left.\left.-\left(k / p^{2}\right)(\epsilon-p \cos \theta)(5 \epsilon+p k \cos \theta)\right]\right\},
\end{aligned}
$$

where $\alpha \approx 1 / 137$ is the fine-structure constant, $r_{0}$ is the classical electron radius, and $p_{2}^{*}=\sqrt{\epsilon_{2}^{* 2}-1}$ is the momentum of the final electron in the rest frame of the proton.

It is instructive to compare the differential cross section (18) with that of the normal electron-proton bremsstrahlung where the electron has the same velocity, i.e., $\epsilon_{1}^{*}=\epsilon$. One notes the following: at low proton energies the behaviour of the two cross sections is similar. If the energies become higher, this changes drastically. The electron-proton cross section has a sharp maximum at small angles originating from the denominator $k^{*}\left(\epsilon_{1}^{*}-p_{1}^{*} \cos \theta^{*}\right)$. Passing on to the rest frame of the proton, this expression transforms to $k$ so that the proton-electron cross section becomes more isotropic, except for $k \approx k_{\max }$. In any case protons can emit photons of higher energy than electrons. For instance, protons of kinetic energy $E=1 \mathrm{GeV}(v \approx 0.875 c)$ produce photons of maximum energy $h v_{\max } \approx 2.105 \mathrm{MeV}$, whereas electrons with the same velocity $\left(E_{1} \approx 545 \mathrm{keV}\right)$ generate photons with $h v<E_{1}$.

The spectrum integrated over the photon angles is given by

$$
\begin{aligned}
\frac{\mathrm{d} \sigma}{\mathrm{d} k} & =\int \frac{1}{\epsilon-p \cos \theta} \frac{\mathrm{d}^{2} \sigma^{*}}{\mathrm{~d} k^{*} \mathrm{~d} \Omega^{*}} \mathrm{~d} \Omega \\
& =2 \pi \int_{\cos \theta_{0}}^{1} \frac{1}{\epsilon-p \cos \theta} \frac{\mathrm{d}^{2} \sigma^{*}}{\mathrm{~d} k^{*} \mathrm{~d} \Omega^{*}} \mathrm{~d}(\cos \theta) .
\end{aligned}
$$

The lower integration limit is obtained from (6), setting $\epsilon_{1}=1$, $p_{1}=0$ :

$\cos \theta_{0}$

$$
= \begin{cases}-1 & \text { for } k \leq \frac{\epsilon-1}{\epsilon+p+m_{\mathrm{e}} / m_{\mathrm{p}}}, \\ \frac{\epsilon(k-1)+1+\left(m_{\mathrm{e}} / m_{\mathrm{p}}\right) k}{p k} & \text { for } \frac{\epsilon-1}{\epsilon+p+m_{\mathrm{e}} / m_{\mathrm{p}}} \leq k \leq \frac{\epsilon-1}{\epsilon-p+m_{\mathrm{e}} / m_{\mathrm{p}}} .\end{cases}
$$

\footnotetext{
${ }^{2}$ It is important to notice that the photon emission angle in the proton rest system is $\pi-\theta^{*}$.
}

Applying the cross section (18), nearly all of the integrations in (19) can be performed analytically. According to (20) one has to distinguish between two cases:

$$
\text { a) } \begin{aligned}
& \frac{\epsilon-1}{\epsilon+p+m_{\mathrm{e}} / m_{\mathrm{p}}} \leq k \leq \frac{\epsilon-1}{\epsilon-p+m_{\mathrm{e}} / m_{\mathrm{p}}}: \\
& \frac{\mathrm{d} \sigma_{1}}{\mathrm{~d} k}=\frac{\alpha r_{0}^{2}}{p^{2} k}\left\{W _ { 1 } ( \epsilon - p ) \left[-\frac{5 \epsilon}{12 k^{3}}-\frac{\epsilon-p}{4 k^{2}}+\frac{4 \epsilon}{3 k^{2}}\right.\right. \\
&-\frac{\epsilon}{p^{2} k}\left(p^{2}+\frac{1}{3} \epsilon p+\frac{1}{2}\right)+\frac{\epsilon+p}{2 p^{2}}\left(1 / p^{2}-\frac{1}{3}\right)-\frac{2}{3} k(\epsilon-p) \\
&\left.+\frac{k}{6 p^{2}}(\epsilon+p)\right] \\
&+L_{1}(\epsilon-p)\left[\frac{2 \epsilon^{2}+3}{12 k^{3}}-\frac{2 \epsilon^{2}}{3 k^{2}}+\frac{8 \epsilon^{2}}{3 k}+\frac{1}{6 k}+\frac{1}{2}-\frac{1}{2 k-1}\right] \\
&-\frac{L_{2}(\epsilon-p)}{p^{3}}\left[p^{3}+\left\{2 \epsilon p+\left(\epsilon^{2}+p^{2}\right) k\right\}(\epsilon-p)\right. \\
&-\epsilon k\left(2 \epsilon^{2}+\frac{3}{2}+3 / p^{2}+\frac{1}{2} k\right)(\epsilon-p)^{2} \\
&+\frac{1}{3}\left\{2 \epsilon^{2}+4\left(\epsilon^{2} k / p^{2}\right)\left(\epsilon^{2}+2\right)+\left(\epsilon^{2}+p^{2}\right) k^{2}\right\}(\epsilon-p)^{3} \\
&\left.-\frac{\epsilon k}{2 p^{2}}\left(\epsilon^{2}+2\right)(\epsilon-p)^{4}\right]+\frac{2 k}{2 k-1} L_{3}(\epsilon-p) \\
&\left.+2 \int_{\epsilon-p}^{x_{0}}\left[(1-k) \frac{L_{3}(x)}{W_{2}(x)}-\frac{L_{1}(x)}{x}\right] \mathrm{d} x\right\},
\end{aligned}
$$

where

$$
\begin{aligned}
& W_{1}(x)=\sqrt{p^{2}-2 \epsilon k x+k^{2} x^{2}}, \\
& W_{2}(x)=\sqrt{p^{2}-2 \epsilon k x+2 k+k^{2} x^{2}}, \\
& L_{1}(x)=\ln \left(\epsilon-k x+W_{1}\right), \quad L_{2}(x)=\ln \frac{p^{2}-\epsilon k x+p W_{1}}{k x}, \\
& L_{3}(x)=\ln \frac{W_{1}+W_{2}}{\sqrt{2 k}}, \\
& x_{0}=\epsilon-p \cos \theta_{0}=\frac{\epsilon-1}{k}-\frac{m_{\mathrm{e}}}{m_{\mathrm{p}}} .
\end{aligned}
$$

In deriving the expression (21) the approximations

$W_{1}\left(x_{0}\right)=L_{1}\left(x_{0}\right)=L_{2}\left(x_{0}\right)=L_{3}\left(x_{0}\right)=0$

were made. The exact value would be, e.g.,

$W_{1}\left(x_{0}\right)=\sqrt{k\left(m_{\mathrm{e}} / m_{\mathrm{p}}\right)\left(2+k m_{\mathrm{e}} / m_{\mathrm{p}}\right)}$,

which is a small quantity for $k \ll m_{\mathrm{p}} / m_{\mathrm{e}} \approx 1836$. The nonvanishing magnitude of $W_{1}\left(x_{0}\right), L_{1}\left(x_{0}\right)$, etc. arises from the fact, that the proton recoil is neglected in the cross section (18), whereas it was allowed for in the kinematics, resulting in the additional term $-m_{\mathrm{e}} / m_{\mathrm{p}}$ of $x_{0}$. If the recoil would also be taken into account in the cross section, as was performed by Drell (1952), the relations (26) would be satisfied exactly.

In the case $k=\frac{1}{2}$ one has to take the limit

$$
\begin{aligned}
& \lim _{k \rightarrow 0.5}\left\{\frac{1}{2 k-1}\left[2 k L_{3}(\epsilon-p)-L_{1}(\epsilon-p)\right]\right\} \\
& =\ln \left\{\frac{1}{2}\left[\epsilon+p+\sqrt{(\epsilon+p)^{2}-4}\right]\right\}-\frac{1}{2} \sqrt{1-4(\epsilon-p)^{2}} .
\end{aligned}
$$


b) $k \leq \frac{\epsilon-1}{\epsilon+p+m_{\mathrm{e}} / m_{\mathrm{p}}}$ :

$$
\begin{aligned}
\frac{\mathrm{d} \sigma_{2}}{\mathrm{~d} k}= & \frac{\alpha r_{0}^{2}}{p^{2} k}\left\{W _ { 1 } ( \epsilon + p ) \left[\frac{5 \epsilon}{12 k^{3}}+\frac{\epsilon+p}{4 k^{2}}-\frac{4 \epsilon}{3 k^{2}}\right.\right. \\
& +\frac{\epsilon}{p^{2} k}\left(p^{2}-\frac{1}{3} \epsilon p+\frac{1}{2}\right)+\frac{\epsilon-p}{2 p^{2}}\left(\frac{1}{3}-1 / p^{2}\right) \\
& \left.+\frac{2}{3}(\epsilon+p) k-\frac{k}{6 p^{2}}(\epsilon-p)\right]-L_{1}(\epsilon+p)\left[\frac{2 \epsilon^{2}+3}{12 k^{3}}-\frac{2 \epsilon^{2}}{3 k^{2}}\right. \\
& \left.+\frac{8 \epsilon^{2}}{3 k}+\frac{1}{6 k}+\frac{1}{2}-\frac{1}{2 k-1}\right] \\
& +\frac{L_{2}(\epsilon+p)}{p^{3}}\left[-p^{3}+\left\{\left(\epsilon^{2}+p^{2}\right) k-2 \epsilon p\right\}(\epsilon+p)\right. \\
& -\epsilon k\left(2 \epsilon^{2}+\frac{3}{2}+3 / p^{2}+\frac{1}{2} k\right)(\epsilon+p)^{2} \\
& +\frac{1}{3}\left\{2 \epsilon^{2}+4\left(\epsilon^{2} k / p^{2}\right)\left(\epsilon^{2}+2\right)+\left(\epsilon^{2}+p^{2}\right) k^{2}\right\}(\epsilon+p)^{3} \\
& \left.-\frac{\epsilon k}{2 p^{2}}\left(\epsilon^{2}+2\right)(\epsilon+p)^{4}\right]+\frac{2 k}{2 k-1} L_{3}(\epsilon+p) \\
& \left.+2 \int_{x_{0}}^{\epsilon+p}\left[(1-k) \frac{L_{3}(x)}{W_{2}(x)}-\frac{L_{1}(x)}{x}\right] \mathrm{d} x\right\}+\frac{\mathrm{d} \sigma_{1}}{\mathrm{~d} k} .
\end{aligned}
$$

For small values of the photon energy, $k \ll p^{2} / \epsilon^{2}$, it is convenient to expand the cross section (29) into powers of $k$, in order to avoid rounding errors. Then the integrals can be carried out and the terms with negative powers of $k$ cancel. Up to relative order $k^{2}$ we get

$$
\begin{aligned}
\frac{\mathrm{d} \sigma}{\mathrm{d} k} \approx & \frac{\alpha r_{0}^{2}}{p^{2} k}\left\{\frac{16}{3} \epsilon^{2}\left(1-k+k^{2}\right) \ln \left(2 p^{2} / k\right)-4 \ln ^{2}(\epsilon+p)\right. \\
& -4 \epsilon\left[p+\epsilon^{4} /\left(3 p^{3}\right)\right] \ln (\epsilon+p)+\frac{28}{9} \epsilon^{2}+4 /\left(3 p^{2}\right) \\
& +k\left[\frac{\epsilon}{3 p}\left(16 p^{2}+2-\frac{2}{p^{2}}+\frac{3}{p^{4}}\right) \ln (\epsilon+p)-\frac{46}{9} \epsilon^{2}\right. \\
& \left.-\frac{5}{p^{2}}-\frac{1}{p^{4}}\right]+k^{2}\left[\frac{128}{45} \epsilon^{2}+\frac{1307}{45}-\frac{5}{p^{2}}-\frac{4}{p^{4}}\right. \\
& \left.\left.-\frac{\epsilon}{3 p}\left(16 p^{2}+6-1 / p^{2}\right) \ln (\epsilon+p)\right]\right\} .
\end{aligned}
$$

The relative error of this formula is less than $0.3 \%$ for kinetic energies of the proton $E>100 \mathrm{MeV}$ and $k<0.03$, or $E>$ $1 \mathrm{GeV}$ and $k<0.07$.

In the cross section (18) the distortion of the electron wave functions by the proton's Coulomb field is neglected. This can be taken into account approximately if (18) is multiplied by the Elwert factor (Elwert 1939; Elwert \& Haug 1969)

$F_{E}=\frac{a_{2}}{a_{1}} \frac{1-\mathrm{e}^{-2 \pi a_{1}}}{1-\mathrm{e}^{-2 \pi a_{2}}}$,

where $a_{1}=\alpha \epsilon / p$ and $a_{2}=\alpha \epsilon_{2}^{*} / p_{2}^{*}$. In particular, this results in a non-vanishing cross section at the short-wavelength limit of the bremsstrahlung process. Then, however, the integration in (19) has to be performed numerically. The cross sections given by Haug (1972) were calculated in this way. The effect of the Coulomb correction is appreciable only near the high-energy limit of the PEB spectrum.

\section{Photon spectra from protons with power-law spectra}

\subsection{Thin target}

In a thin target the energy distribution of the incident protons is not influenced by collisions with other particles. Denoting the spectral flux of the protons by $J_{\mathrm{p}}(\epsilon)$, the PEB photon spectrum (number of photons emitted per second, $\mathrm{cm}^{3}$, and $\mathrm{MeV}$ ) in a plasma with the ambient electron density $n_{\mathrm{e}}\left(\mathrm{cm}^{-3}\right)$, assumed to be uniform, is given by

$N(k)=\left(m_{\mathrm{p}} / m_{\mathrm{e}}\right) n_{\mathrm{e}} \int_{\epsilon_{m}}^{\infty} J_{\mathrm{p}}(\epsilon) \frac{\mathrm{d} \sigma}{\mathrm{d} k} \mathrm{~d} \epsilon$.

The lower integration limit $\epsilon_{m}=\epsilon_{\min }(k)$ is given by Eq. (10) and the factor $m_{\mathrm{p}} / m_{\mathrm{e}}$ originates from the different energy units of $\epsilon$ and $k$.

In many astrophysical applications $J_{\mathrm{p}}(\epsilon)$ has the form of a power law in the kinetic energy with spectral index $\delta$,

$J_{\mathrm{p}}(\epsilon)=J_{0}(\epsilon-1)^{-\delta} \mathrm{cm}^{-2} \mathrm{~s}^{-1} \mathrm{MeV}^{-1}$,

where $J_{0}$ is a constant characterizing the total flux. Then the photon spectrum is given by

$N(k)=\left(m_{\mathrm{p}} / m_{\mathrm{e}}\right) J_{0} n_{\mathrm{e}} \int_{\epsilon_{m}}^{\infty}(\epsilon-1)^{-\delta} \frac{\mathrm{d} \sigma}{\mathrm{d} k} \mathrm{~d} \epsilon$.

In nonrelativistic approximation the integration in (34) can be performed analytically. One gets, analogous to normal bremsstrahlung (Brown 1971),

$N_{\mathrm{nr}}(k)=\frac{8}{3} \alpha r_{0}^{2}\left(m_{\mathrm{p}} / m_{\mathrm{e}}\right)\left(n_{\mathrm{e}} J_{0} / \delta\right) B\left(\delta, \frac{1}{2}\right) k^{-(\delta+1)}$,

where $B(x, y)$ denotes the beta function.

The upper curve of Fig. 2 shows the photon energy distribution for the spectral index $\delta=2.6$ of the cosmic-ray protons. At low photon energies $k<0.07$, corresponding to $h v<36 \mathrm{keV}$, the spectrum has also the form of a power law with spectral in$\operatorname{dex} \gamma=\delta+1=3.60$, in agreement with Eq. (35). If $k$ increases, the photon spectrum flattens due to relativistic effects, and the spectral index becomes $\gamma=2.68$ for $k>10$ or $h v>5.1 \mathrm{MeV}$. Inclusion of the Coulomb correction factor (31) would change the photon flux a little at low energies $k$. However, the slope of the spectrum is virtually the same.

\subsection{Thick target}

In a thick target the impinging protons are slowed down by collisions with ambient particles. Employing normal energy units, the number of photons of energy $h v$ emitted by protons with the initial kinetic energy $E_{0}$ is

$$
\begin{aligned}
Z(h v) & =n_{\mathrm{e}} \int_{E=E_{0}}^{E=E_{\min }} \frac{\mathrm{d} \sigma}{\mathrm{d}(h v)} v \mathrm{~d} t \\
& =n_{\mathrm{e}} \int_{E_{0}}^{E_{\min }} \frac{\mathrm{d} \sigma}{\mathrm{d}(h v)} v\left(\frac{\mathrm{d} E}{\mathrm{~d} t}\right)^{-1} \mathrm{~d} E,
\end{aligned}
$$

where $v$ is the instantaneous proton velocity and $\mathrm{d} E / \mathrm{d} t$ is the energy loss rate of the protons in the plasma. Designating the 
proton injection rate (protons per $\mathrm{cm}^{2}, \mathrm{~s}$, and $\mathrm{MeV}$ ) by $f\left(E_{0}\right)$ and the target area by $A$, the photon production rate is given by

$$
\begin{aligned}
N(h v) & =A \int_{E_{\min }}^{\infty} Z(h v) f\left(E_{0}\right) \mathrm{d} E_{0} \\
& =A n_{\mathrm{e}} \int_{E_{\min }}^{\infty} \mathrm{d} E_{0} f\left(E_{0}\right) \int_{E_{\min }}^{E_{0}} \mathrm{~d} E \frac{\mathrm{d} \sigma}{\mathrm{d}(h v)} v(E)\left|\frac{\mathrm{d} E}{\mathrm{~d} t}\right|^{-1} .
\end{aligned}
$$

Switching to the energy units $\epsilon$ and $k$, this has the form

$$
\begin{aligned}
N(k) & =A \frac{m_{\mathrm{p}}}{m_{\mathrm{e}}} n_{\mathrm{e}} c \int_{\epsilon_{\min }(k)}^{\infty} \mathrm{d} \epsilon_{0} f\left(\epsilon_{0}\right) \int_{\epsilon_{\min }(k)}^{\epsilon_{0}} \mathrm{~d} \epsilon \frac{p}{\epsilon}\left|\frac{\mathrm{d} \epsilon}{\mathrm{d} t}\right|^{-1} \frac{\mathrm{d} \sigma}{\mathrm{d} k} \\
& =A \frac{m_{\mathrm{p}}}{m_{\mathrm{e}}} n_{\mathrm{e}} c \int_{\epsilon_{\min }(k)}^{\infty} \mathrm{d} \epsilon \frac{p}{\epsilon}\left|\frac{\mathrm{d} \epsilon}{\mathrm{d} t}\right|^{-1} \frac{\mathrm{d} \sigma}{\mathrm{d} k} \int_{\epsilon}^{\infty} \mathrm{d} \epsilon_{0} f\left(\epsilon_{0}\right) .
\end{aligned}
$$

If we choose again a power law for the proton injection rate,

$f\left(\epsilon_{0}\right)=I_{0}\left(\epsilon_{0}-1\right)^{-\delta}$,

the second integral of (38) can be easily solved yielding

$$
\begin{aligned}
N(k)= & I_{0} A \frac{m_{\mathrm{p}}}{m_{\mathrm{e}}} n_{\mathrm{e}} c \int_{\epsilon_{\min }(k)}^{\infty} \mathrm{d} \epsilon \frac{p}{\epsilon}\left|\frac{\mathrm{d} \epsilon}{\mathrm{d} t}\right|^{-1} \frac{\mathrm{d} \sigma}{\mathrm{d} k} \\
& \times \int_{\epsilon-1}^{\infty} \mathrm{d}\left(\epsilon_{0}-1\right)\left(\epsilon_{0}-1\right)^{-\delta} \\
= & \frac{I_{0} A m_{\mathrm{p}} n_{\mathrm{e}} c}{(\delta-1) m_{\mathrm{e}}} \int_{\epsilon_{\min }(k)}^{\infty} \frac{\sqrt{\epsilon^{2}-1}}{\epsilon}\left|\frac{\mathrm{d} \epsilon}{\mathrm{d} t}\right|^{-1} \\
& \times \frac{\mathrm{d} \sigma}{\mathrm{d} k}(\epsilon-1)^{-(\delta-1)} \mathrm{d} \epsilon .
\end{aligned}
$$

Considering protons impinging on a hydrogen target with number density $n_{\mathrm{H}}$, the energy loss rate is given by

$\frac{\mathrm{d} \epsilon}{\mathrm{d} t}=-4 \pi r_{0}^{2} n_{\mathrm{H}} \frac{m_{\mathrm{e}} c^{2}}{m_{\mathrm{p}} v} \Lambda_{\mathrm{p}}$,

where $v=(p / \epsilon) c$ is the proton velocity. The Coulomb logarithm $\Lambda_{p}$ is different for plasmas consisting of neutral or ionized hydrogen (Emslie 1978).

The thick-target photon spectrum plotted in Fig. 2 was calculated for a completely ionized hydrogen target with electron number density $n_{\mathrm{e}}$ using the energy loss rate (Lang 1980)

$\frac{\mathrm{d} \epsilon}{\mathrm{d} t}=-2 \pi r_{0}^{2} n_{\mathrm{e}} \frac{m_{\mathrm{e}} c^{2}}{m_{\mathrm{p}} v}\left\{\ln \left(\frac{m_{\mathrm{e}}^{2} v^{2} W}{2 \pi \alpha \hbar^{3} c n_{\mathrm{e}}}\right)+1-2 \frac{v^{2}}{c^{2}}\right\}$,

where

$W=\frac{2 p^{2}}{2\left(m_{\mathrm{e}} / m_{\mathrm{p}}\right) \epsilon+1} m_{\mathrm{e}} c^{2}$

is the maximum kinetic energy transferred to free electrons. To facilitate the comparison with the thin-target curve the spectral index was again chosen $\delta=2.6$ (the magnitude of the photon fluxes is arbitrary). At very low energies the photon spectrum can be approximated by a power law with $\gamma \approx \delta-1=1.6$, again in agreement with the nonrelativistic result of Brown (1971). With increasing $k$ it steepens continuously and the spectral

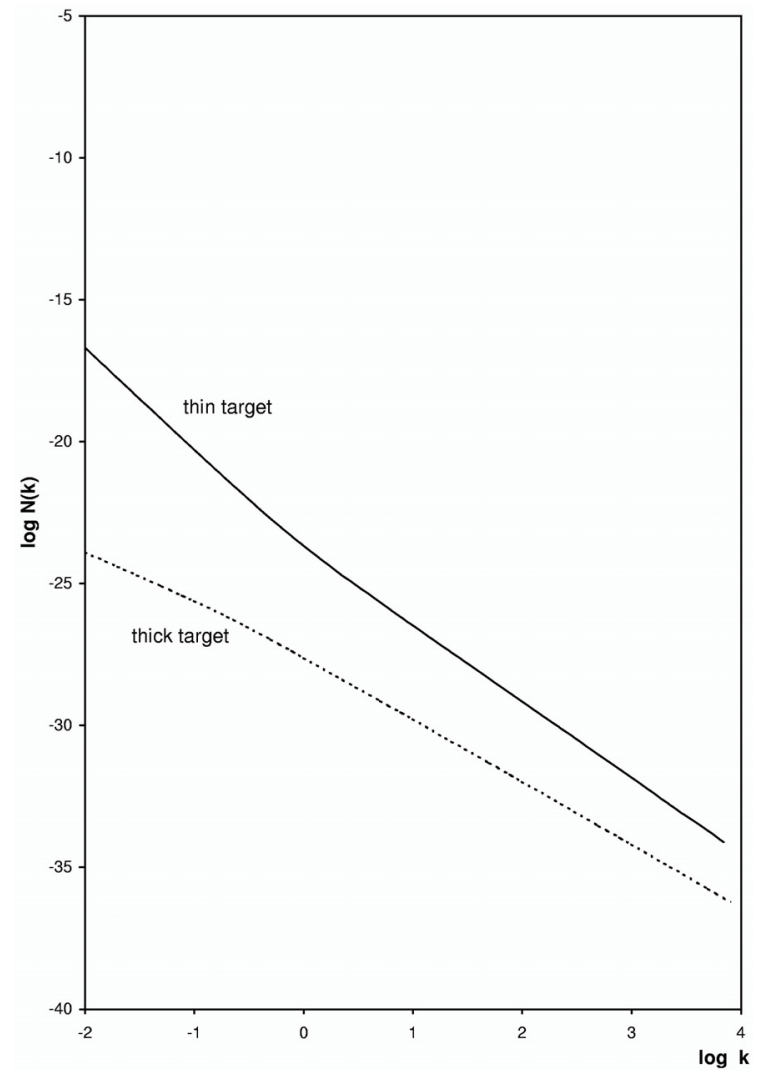

Fig. 2. PEB photon spectra from protons with a power-law spectrum, spectral index $\delta=2.6$, for thin (upper curve) and thick targets. The proton fluxes are arbitrary.

index takes the value $\gamma \approx 2.23$ at $k=10^{4}$. That is, even though the spectral indexes approach each other in the thick and thintarget cases, the thick-target PEB spectra are flatter in the whole energy range.

\section{References}

Baring, M. G., Jones, F. C., \& Ellison, D. C. 2000, ApJ, 528, 776

Boldt, E., \& Serlemitsos, P. 1969, ApJ, 157, 557

Brown, J. C. 1971, Sol. Phys., 18, 489

Brown, R. L. 1970, Lett. Nuovo Cimento, 4, 941

Dogiel, V. A., Ichimura, A., Inoue, H., \& Masai, K. 1998, PASJ, 50, 567

Drell, S. D. 1952, Phys. Rev., 87, 753

Elwert, G. 1939, Ann. Phys. (Leipzig), 34, 178

Elwert, G., \& Haug, E. 1969, Phys. Rev., 183, 90

Emslie, A. G. 1978, ApJ, 224, 241

Emslie, A. G., \& Brown, J. C. 1985, ApJ, 295, 648

Haug, E. 1972, Astrophys. Lett., 11, 225

Haug, E. 1997, A\&A, 326, 417

Heristchi, D. 1986, ApJ, 311, 474

Jones, F. C. 1971, ApJ, 169, 503

Lang, K. R. 1980, Astrophysical Formulae, 2nd edition (Berlin: Springer-Verlag)

Pohl, M. 1998, A\&A, 339, 587

Sauter, F. 1934, Ann. Phys. (Leipzig), 20, 404

Valinia, A., \& Marshall, F. E. 1998, ApJ, 505, 134 